\title{
Intelligent Messages in Bacterial DNA - a Sequel to SETI?
}

\author{
N. Chandra Wickramasinghe ${ }^{1,2,3,4}$, Gensuke Tokoro ${ }^{4}$ and Robert Temple ${ }^{5}$ \\ ${ }^{1}$ Buckingham Centre for Astrobiology, University of Buckingham, UK \\ ${ }^{2}$ Centre for Astrobiology, University of Ruhuna, Matara, Sri Lanka \\ ${ }^{3}$ National Institute of Fundamental Studies, Kandy, Sri Lanka \\ ${ }^{4}$ Institute for the Study of Panspermia and Astroeconomics, Gifu, Japan \\ ${ }^{5}$ History of Chinese Science and Culture Foundation Conway Hall, London, UK \\ Email: ncwick@gmail.com
}

\begin{abstract}
It is proposed that the future trajectory of SETI (Search for Extraterrestrial Intelligence) and our exploration of alien planets and alien intelligence could be to consider the possibility of receiving and transmitting coded messages embedded as DNA inserts in bacteria and/or viruses. Physical space-travel and ambitions of space colonisation may well give way to a new era of "cultural" microbial colonisation of our galaxy.
\end{abstract}

Keywords: SETI, panspermia, coded DNA, cultural colonisation

\section{Introduction}

Where is the evidence of alien intelligent life? If such aliens exist in great abundance outside the Earth why have we not found them? These questions constitute the well-known Fermi paradox named after the nuclear physicist Enrico Fermi (1). Early discussions have tended to err on the side of the possible uniqueness of Homo sapiens as an intelligent species capable, or nearly capable of, extensive space travel and space colonisation. However, more recently this position has been challenged by the discoveries of habitable exoplanets leading to estimates of their total numbers running into tens of billions. Moreover, with growing evidence in support of cometary panspermia (1) as the mode of origin and evolution of life on the Earth and of its transfer, it is becoming increasingly difficult to argue that life will not take root and evolve in self-similar ways on billions of other habitable planets. That subsequent biological evolution would lead up to human-type intelligence and even beyond is also taken as given in such a picture. So, the old Fermi question "Where are they?" now begins to acquire a more powerful resonance.

\section{Space Colonisation}

With a modest extrapolation from the present stage of our space-age technologies to a few decades into the future, travel to a habitable exoplanet a few parsecs away becomes entirely conceivable. However, our relatively short human lifespans $\sim 10^{2}$ years, would necessitate perhaps a couple of reproduction cycles on route to reach a prospective destination outside the solar system. Early colonisation of the East by European nations took place because shipping from England to say India or Sri Lanka took far less than one human generation, months or years at most. The psychological constraints that are entailed in space colonisation that took many generations to reach a destination might make such a project unattractive for us, and likewise also for other human-type civilizations elsewhere in the galaxy. That would be one possible way to wriggle out of the Fermi paradox. They did not come here because it was psychologically inadmissible.

But would an advanced civilization necessarily give up so easily? The desire to make their mark upon a wider galactic community would surely drive them to explore alternative strategies. In order to decide what form such strategies might take it is worth looking at the history of human colonisation of our own planet the Earth. Physical colonisation, that progressed for example in Europe from the fifteenth century onwards, involved travel to distant lands for exploration of the planet as much as for gaining territory and dominance. This often involved a ruthless subjugation and exploitation of other races by colonisers, and in many instances genocide to achieve their goals. Living in the shadows of our own past 
history and attempting even to "decolonise" in the year 2021, another form of colonisation has been shown to be far more desirable. This can be called cultural colonisation or the spreading of soft power from one dominant culture to others. The same process may well be the route that space colonisation will take in the future. The next step might be the exportation of our intellectual, artistic, and ultimately our genetic legacy - our DNA - on a galaxy-wide scale. The physical colonisation of Mars and establishing human colonies there is of course a highly probable first step in the foreseeable future. But beyond that we may need to think of other strategies akin to cultural colonisation to assert our presence and hopefully our dominance and influence.

\section{SETI and Beyond}

If we abandon the aim of physically reaching an alien settlement located many light years away, the next option would be to make contact through information transfers in various ways. This was the raison d'etre for the development of the SETI programmes that were initiated in the 1960's. The results of SETI so far have been disappointing, although the recent revival of the project with vast injections of funds by Russian billionaire Yuri Milna may possibly change the outcome in some degree.

However, it is worth exploring the other options that might be available for letting alien civilizations know of our existence, and indeed of our long cultural and technological heritage. Besides sending/receiving coded electromagnetic signals as in the SETI projects, the possibility of distributing physical artefacts of our own civilization is an option that was attempted first with the Pioneer and Voyager spacecraft in the 1970's. The Pioneer 10 and 11 spacecraft, on escape trajectories out of our solar system, carried the famous plaque depicting a sketch defining our location in the galaxy where we are as well as a nude man and woman, just to show what we looked like.

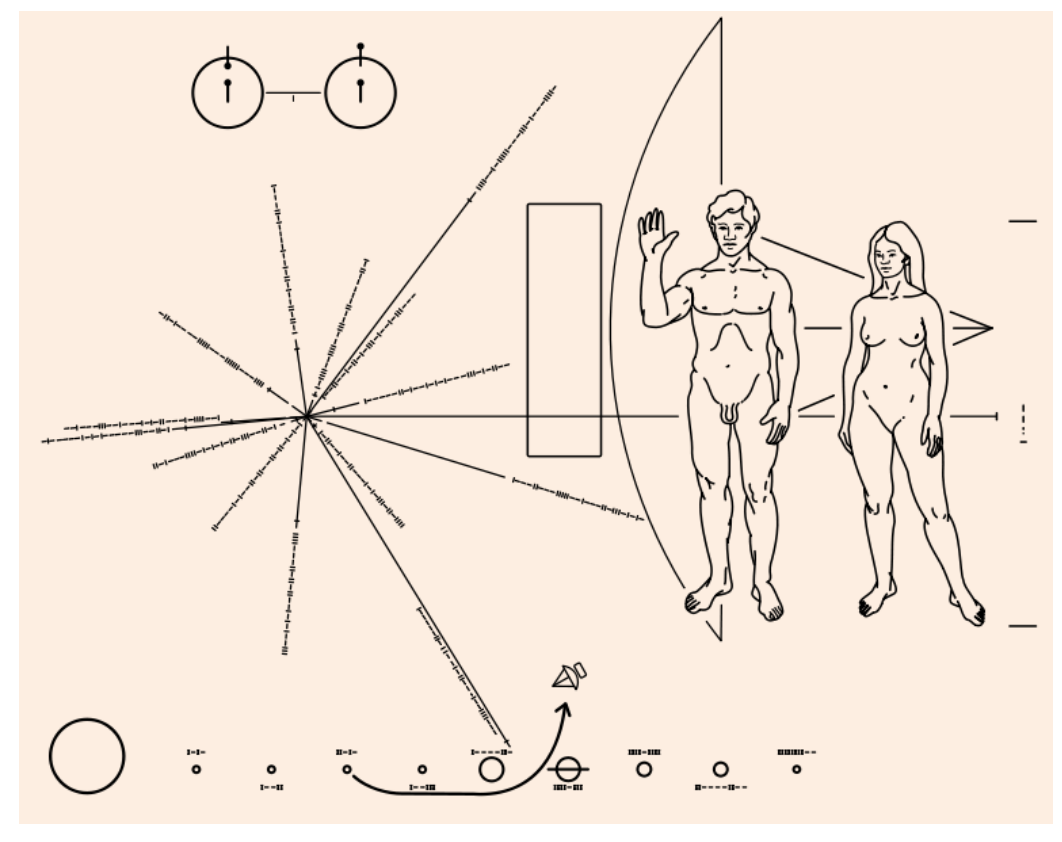

Figure 1. The plaque carried by the Pioneer $10 \& 11$ spacecraft. (Courtesy NASA/JPL)

The later Voyager spacecraft also carried the golden records - a phonograph with "Sounds of the Earth", the reading of which would pose a challenge even for us in the year 2021. A phonograph is already stone-age technology, and later tapes, CD-roms etc are also fast moving into oblivion. 


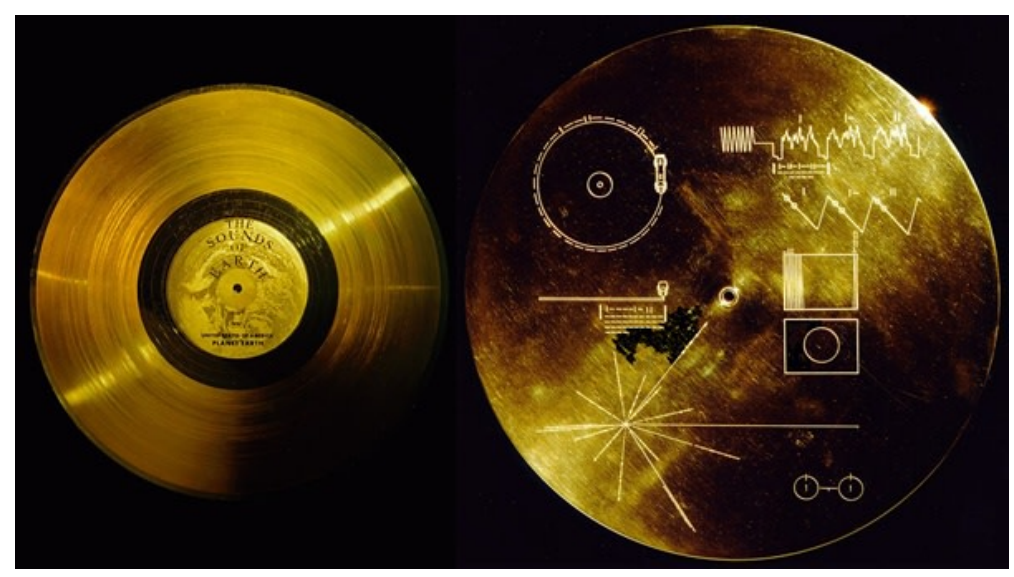

Figure 2. The golden record carried on Voyager spacecraft (Courtesy NASA/JPL)

Next, the New Horizons Mission to Pluto launched in 2006 carried human artefacts including an American flag and most surprisingly the ashes of Clyde Tombaugh, the American astronomer who discovered Pluto in 1930! What a shipwrecked alien coming upon such junk will make of all this is unimaginable! Perhaps William Wordsworth in this Psalm of Life said it all. What we should aim at leaving is more explicitly our intellectual cultural footprints in some indelible, and hopefully universal way, in the "sands of time".

\footnotetext{
"Footprints, that perhaps another, Sailing o'er life's solemn main,

A forlorn and shipwrecked brother, Seeing, shall take heart again."
}

\section{Genetic Messaging}

If cometary panspermia is the explanation for the origin and dispersal of life, then the genetic code will be necessarily universal, and indeed universally known. We also know from modern genetic sequence studies that a considerable fraction of DNA in bacteria, and indeed in higher life forms, comprises noncoding sequences - sequences that are not involved in protein synthesis. These sequences are usually thought of as regulatory sequences, possibly of viral origin. The interest in our present context is that the slipping in of extraneous "coded messages" within silent DNA will not be expected to affect biological function but may well serve as a means for the transfer of digital information. If a message with any desired content can be coded and introduced into a bacterial/viral genome and vast numbers of such a bacterium produced by cloning, it is possible to imagine that this would be the ideal vehicle for the dispersal of the message into space. This is of course strikingly reminiscent of the ideas of directed panspermia first proposed by Francis Crick and Leslie Orgeul (3) (Icarus, 19(3), 341, 1973) who suggested that life itself was introduced to Earth deliberately by an advanced alien civilization.

The potential of bacterial DNA to carry a non-coding insert with a vast amount of digital data has recently been demonstrated by Erlich and Zielinski (4) (2017) (Y. Erlich and D. Zielinski, 2017. Science, $355,950)$. In what Elrich and Zielinski described as a DNA fountain, they demonstrated the feasibility of storing and retrieving a vast amount of digital data (terabytes), including movie files, from a single microgramme of DNA. This new technology opens up the possibility of sending complex messages to alien civilizations using coded DNA within bacteria and viruses, although the problem of coding and decoding still remains to be addressed.

More recent studies by Organick et al (2020) (5) have further demonstrated the astounding potential of synthetic DNA to be deployed as a storage medium for astronomically vast quantities of archival data. The storage of such data in digital form, followed by a reliable file recovery system using standard PCR (polymer chain reaction) techniques, has now been amply confirmed with new results showing almost two orders of magnitude advance on earlier studies. Although the stable storage and retrieval of vast quantities of data has now been demonstrated and would serve as a means of broadcasting information 
to extraterrestrial civilizations, the presumption that they would be in possession of the same means of coding/decoding information including PCR-type technologies is an assumption that might be questioned. We could argue that our human technologies must in some sense be regarded as universal with a convergence to a unique coding/decoding system as we use on Earth, but this would necessarily remain an unproved speculation at the present time.

We can imagine a substantial load (perhaps tonnes) of message-carrying bacteria to be produced in a biotech laboratory on the Earth and released outside the Earth's gravity over the period of, say, one solar year - i.e a single revolution of our planet around the sun. For a bacterial clump of radius $\sim 1$ micrometre released at 1 AU (Earth's orbital distance) from the sun the ratio of radiation pressure to solar gravity $(\mathrm{P} / \mathrm{G})$ can be shown to exceed unity (typically, we estimate $\mathrm{P} / \mathrm{G}=1.5$ ), and the speed of escape $\mathrm{V}$ from the outer edge of the solar system can then be calculated as

$$
V=\sqrt{\frac{2 \alpha k M}{R}}
$$

where $\alpha=\mathrm{P} / \mathrm{G}-1 \approx 0.5$ for a micron-sized particle, $\mathrm{k}$ is the universal gravitational constant, $\mathrm{M}$ is the solar mass and R is the Earth's heliocentric distance of $1 \mathrm{AU}$ (see eg Wickramasinghe et al, (6)). Inserting appropriate values for these quantities we find that speed of leaving the solar system is $3 \times 10^{6} \mathrm{~cm} / \mathrm{s}$ and the distance of a "wave" of message-carrying bacteria reaching a distance of $10 \mathrm{pc}$ is only $\sim 0.3 \mathrm{My}$. This is a relatively short time in our own evolutionary history as an intelligent species on Earth. Over such a timescale we can estimate that the total dose of ionising radiation (low energy Cosmic Rays) is 10Mrad, a dose that may be expected to reduce viability of a typical bacterium by millions, but one which would be withstood by radiation resistant bacterial species such as M.radiodurans. In any case even reduction of viability by millions will not be a problem because the numbers that are expelled could be vast. The situation could well be similar to the sowing of seeds in the wind - few are destined to survive but so many are the seeds that some among them must surely survive.

\section{$5 \quad$ Are They Here Already?}

Finally, we return to the Fermi question "Where are they?" And the answer may already lie unnoticed in our own backyard (7,8). This possibility was already noted by Zubrin (9) who speculated that interstellar messages might lie concealed within the vast numbers of cosmic bacteria that are most probably in our midst. We have noted that our own studies over the past 2 decades (which are still continuing) have recovered bacteria which we have argued are falling through the atmosphere from space. Could the long-awaited alien messages already lie concealed within them waiting to be discovered as suggested by Zubrin (9)? Our estimates of the bacterial influx to the Earth lead to amounts that cannot be ignored. We have estimated the total bacterial influx to be in the region of 3 tonnes per day over the whole Earth, amounting to a DNA input of $3 \times 10^{-3}$ tonnes per day. The intelligent "message in the bottle" from our ET neighbours may be lurking within the non-coding fraction of this daily bacterial input of DNA from space. To examine this data exhaustively may be time-consuming and expensive, but perhaps not so compared with the cost of other SETI programmes that are being planned for the near future.

Acknowledgement. We are grateful to Professor Michael Smith for useful comments and criticisms

\section{References}

1. Hart, Michael H. 1975. Explanation for the Absence of Extraterrestrials on Earth, Quarterly Journal RAS, 16, 128.

2. Hoyle, F. \& Wickramasinghe, N.C. 1981. Comets - A vehicle for panspermia, in Comets and the origin of life (ed. C. Ponnamperuma) D. Reidel (reprinted as Astrophys. Space Sci., 268, 333, 1999).

3. Crick, F. H.; Orgel, L. E. (1973). "Directed panspermia". Icarus. 19 (3): 341-346.

4. Erlick, Y. and Zielinski, D., 2017. DNA fountain enables robust and efficient storage architecture, Science, 355, 950 
5. Organick, L., Chen, Y., Dumas Ang, S. et al. 2020. Probing the physical limits of reliable DNA data retrieval. Nat Commun 11, 616 https://doi.org/10.1038/s41467-020-14319-8

6. Wickramasinghe, J., Wickramasinghe, N.C., and Napier, W., 2010. Comets and the Origin of Life (World Scientific Publisher, Singapore)

7. Wickramasinghe, N.C., Maganarachchi, D., Temple, R. et al. 2020, The Search for Bacteria and Viruses in the Stratosphere, Ad.Ap.5(2)

8. Harris, M.J., Wickramasinghe, N.C., Lloyd, D., Narlikar, J.V., Rajaratnam, P., Turner, M.P., Al-Mufti, S., Wallis, M.K. \& Hoyle, F. 2001. The detection of living cells in stratospheric samples, Proc SPIE 4495, $192-198$.

9. Zubrin, R., 2017. Interstellar Communication Using Microbial Data Storage: Implications for SETI, J.British Interplanetary Soc. 70, 163-174 